$16^{\text {th }}$ International Congress of Metrology, 06014 (2013)

DOI: $10.1051 /$ metrology/201306014

(c) Owned by the authors, published by EDP Sciences, 2013

\title{
Traceable flatness calibration with substitution method
}

Tero Ristonen ${ }^{1, \mathrm{a}}$, Paul H. Andersson ${ }^{1}$ and Heikki Tikka

${ }^{1}$ Tampere University of Technology, Department of Production Engineering, P.O.Box 589, 33101 Tampere, Finland

\begin{abstract}
This paper describes a traceable method to calibrate a flat surface with substitution method using a CMM within the manufacturing unit. Method has been tested in an accredited calibration laboratory with a high end CMM. Previous publications within academy have described flatness measurement end the uncertainty connected with it very thoroughly, but now the trace to definition of the meter is introduced.
\end{abstract}

\section{Flatness}

Flatness is an attribute that has to be known in order to evaluate whether the object, where the flat is located, is suitable for its purpose of use. Basically in every case when something is connected to other entity, a flatness requirement is needed. The surfaces that are supposed to touch each other after connection must, depending of the case, be tight to prevent leakage, to deliver force or friction or the like. Another, very widely used need for flat surfaces is the need for base for other measuring equipment, such as height gauges. As early as mid 19th century Whitworth [1] published some of his work around generating and manufacturing flat surfaces. Even though the topic as such is old, the need for making research also with it, has never ceased.

\section{Flatness measurement}

Quite many publications about the measurement of flatness have been written. [2-4] are examples from very productive writers that have been publishing often on the measurement of flat surfaces and the uncertainty of the measurement over the years, as have many other researchers as well. They have been working a lot on the measurement strategies involved.

Most common way to measure a flat and afterwards calculate the flatness of it is to carry out the measurements with a grid. The grid consists of straight lines that are measured. The measurements can be made with an autocollimator, laser-interferometer or like in this case with a CMM, just to mention most common methods.

Whatever the method may be, the results are actually information of X-, Y- and Z-co-ordinates of points within lines that has been measured. In figure 1 is drawn the line distribution within the grid in the basic case where number of lines is 8 .

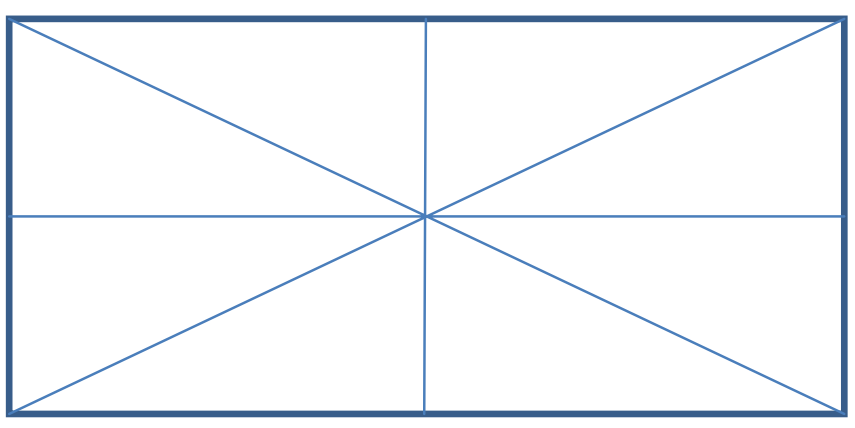

Figure 1. Union Jack layout of the measuring lines, basic case.

The method of measuring and calculating both the flatness and the uncertainty of the measurement is well published. However the trace to international standard of meter is seldom, if at all, mentioned even though whole aspect of flatness is just a derivative of a meter. "The metre is the length of the path travelled by light in vacuum during a time interval of 1/299 792458 of a second." [5]

During this research the measuring method chosen for trial was co-ordinate measurement with a high end CMM. The CMM is located in Tampere University of Technology accredited calibration laboratory K003. The manufacturer of the body of CMM is SIP (SIP CMM5) and the controller is provided by Metrologic (ME5008). Measuring head (PH10MQ) with scanning probe (SP25M) as well as the stylus used is by Renishaw.

CMM is a universal measuring machine that is capable to very wide variety of measuring tasks. CMM is at the date of writing in its mature age and the uncertainty related to measurements with it is quite well known, at least within academy. CMM is also very common measuring device within industry although it is not so well suited for online measurements due to its rather slow operation capability. This can be helped with a visual measuring head such as camera or laser.

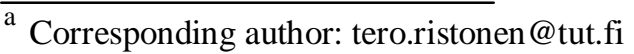




\section{Substitution method}

Widely used calibration technique for determining the trace to definition of the meter is substitution method. Here a known artefact is first measured and the result of unknown workpiece is corrected with calculated data. The measurements must take place right after each other with same styli and operator hopefully with same NCprogram. The last is possible at the time the artefact and workpiece dimensions are very close to each other.

The artefact is calibrated in an accredited calibration laboratory or in a national measurement laboratory and the measurement uncertainty is rather small compared to the measurement uncertainty reachable in the laboratory that uses the result.

International standard ISO 15530-3:2011 [6] describes the similarity requirements between artefact and workpiece. This may bring out the first problem with the method described in this paper. It is often the case that one of the objects under the measurement is made of granite and the other is made of steel. But if the thermal environment is known to sufficient level this does not affect that much. It is said in the requirements that the functional properties are the similarity condition not necessary the actual properties.

\section{New principle}

The principle introduced here is to use calibrated straight edge or a sine bar as a calibrated artefact to bring the trace to the CMM. During this research a straight edge calibrated in national measurement institute "MIKES" was used. The artefact is measured in the same positions that the plane to be calibrated will be measured. The error that is calculated in the artefact measurement can be corrected when calculating the flatness of the plane. The high end CMM used does not have that much straightness error that compensation would be possible. The method will be verified further when a CMM that has significant straightness error is introduced to writers and they have possibility to use it also for this purpose.

During the research software was programmed with MATLAB to do the flatness calculations based on line wise measured point data, but the software does not differ that much from software introduced in [3], [4] or other methods published and therefore will not be reported in detail here. All the software known to authors, create a straight line from the measured points. The data from several (basic case eight) lines are added to form a plane. After this a manipulation of the lines (points) is calculated to form the plane. Calculations are based on the least squares or minimum zone principle. Former minimizes the quadratic error while the latter calculates an ideal line from the measured results in such a way that maximum deviation between the ideal and the actual measurement is the least possible.[7]

In [4] Gusel et al wrote: "Reliability of the used measurement equipment is one of the main factors that affect the reliability of the measurement and its results". Taking into account this and also the fact that no measurement result is informative, or strongly said even valid, without measurement uncertainty we must think the case from the perspective of measurement uncertainty. Measurement uncertainty is described and gone through with examples in [8], [9]

The standard uncertainty can be calculated with equation 1 . In every case single input quantities $\left(\mathrm{u}_{\mathrm{i}}\right)$ must be evaluated according to environment at hand.

$$
u^{2}(y)=\sum_{i=1}^{N} u_{i}^{2}(y)
$$

With little effort one is capable to find reference to uncertainty component evaluation in every special case. The ambient environmental conditions must be evaluated as well as the measured part and measuring equipment influence.

Comparing to other uncertainty calculation procedures presented elsewhere this time also the uncertainty of calibration of the artefact has to be taken into calculations. However this helps measurer to diminish the uncertainty contribution that comes from the measuring device. Overall effect makes calculated measuring uncertainty smaller.

Different strategies with different kind of software algorithms have been introduced to public. In [3], [4], [10] writers have published very thorough calculation examples in their work. Many more works can easily be found.

\section{Conclusion}

The introduction of traceably calibrated reference normal to the calibration of a flat surface with substitution method is a step towards better and more solid grounds for performing other measurements. This leads also to improved product quality on the basis that the flat surfaces form very often ground where afterwards is implemented other manufacturing equipment. In verification of more information about capability of flat surface manufacturing this creates significant value to Finnish, European and also to global manufacturing industry by making it possible to do traceable calibrations of flat surfaces also with factory level CMMs. 


\section{References}

[1] J. Whitworth, Miscellaneous Papers on Mechanical Subjects. (1858)

[2] J. Meijer and C. J. Heuvelman, CIRP Annals Manufacturing Technology, 39, no. 1, pp. 545548, (1990)

[3] H. Haitjema and J. Meijer, European Journal of Mechanical Engineering, 38, no. 4, pp. 165-172, (1993)

[4] A. Gusel, B. Acko, and V. Mudronja, Strojniski Vestnik/Journal of Mechanical Engineering, 55, no. 5, (2009)

[5] BIPM, "Unit of length (metre)," 1983. [Online]. Available:

http://www.bipm.org/en/si/si_brochure/chapter2/ 2-1/metre.html.

[6] International Organization for Standardization (ISO), "Geometrical product specifications (GPS) -- Coordinate measuring machines (CMM): Technique for determining the uncertainty of measurement -- Part 3: Use of calibrated workpieces or measurement standards," 2011.

[7] S. T. Huang, K. Fan, and J. H. H. Wu, Precision Engineering, (15), no. 3, pp. 158-165, (1993)

[8] BIPM - Committee for Guides in Metrology (JCGM 100/WG1), "Evaluation of measurement data - Guide to the expression of uncertainty in measurement," 2008.

[9] EA, "Expression of the Uncertainty of Measurement in Calibration EA-4/02," 1999.

[10] W.-S. Kim and S. Raman, International Journal of Machine Tools and Manufacture, 40, no. 3, pp. 427-443, (2000) 